\title{
Slow magnetic monopoles search in NOvA
}

Alexander Antoshkin, ${ }^{1 *}$ Martin Frank ${ }^{2}$

1 Dzhelepov Laboratory of Nuclear Problems, JINR

${ }^{2}$ University of South Alabama, USA

\begin{abstract}
The NOvA far detector is well suited for finding exotic particles due to its technical features (see [1]). One type of those exotic particles is a "slow" magnetic monopole. It is assumed that the energy deposition of such monopoles should be enough to be registered (see [2]). Measurement of the expected signals was performed on the NOvA test bench at JINR (see [3]). Result of this measurement allows us to perform slow monopole's research using NOvA software and hardware with high efficiency. As a whole, the research can lead to a discovery, or it can limit the existence of monopoles in a wide range of parameters, previously unreachable in other experiments (MACRO, SLIM, RICE, IceCube). Several special software tools have been developed. Slow Monopole Trigger has been created and implemented in the NOvA Data-Driven-Trigger system. Also, an offline reconstruction algorithm has been developed and tested on $5 \%$ of the data. A technical description of these tools and current results of the analysis are presented in this work.
\end{abstract}

\section{INTRODUCTION}

NOvA is a long-baseline neutrino experiment that is studying various parameters of neutrinos (see [4]). The detailed description and specific reports could be found on the project site [5].

The NOvA experiment uses two detectors: a 330 metric-ton near detector at Fermilab and a much larger 14 metric-kiloton far detector in Minnesota just south of the U.S.-Canada border. The detectors are made up of 344,000 cells of extruded, highly reflective plastic PVC filled with liquid scintillator. Light from the scintillator is captured by the fibers and is transmitted to an Avalanche Photodiode (APD). Fibers from 32 cells are grouped on one APD board. Each cell in the far detector measures $3.9 \mathrm{~cm}$ wide, $6.0 \mathrm{~cm}$ deep and 15.5 meters long. The far detector itself measures $15.6 \times 15.6 \times 60$ meters.

Such a large and sensitive detector has never been constructed so close to the Earth's surface. It is a good opportunity to detect lighter monopoles which do not penetrate far into the earth, even with the problem of cosmic background due to the large size of our detector and sufficient accessible angular region (see [6]).

\section{WHY ARE WE INTERESTED IN MONOPOLES?}

Quantum mechanical formulation of the magnetic monopoles was made by Paul Dirac in 1931. Searches for these particles are very important for several reasons:

* Their existence would explain the quantization of electric charge.

* It is possible to restore symmetry between electricity and magnetism by means of their introduction into the theory of electromagnetism.

* Magnetic monopoles naturally appears in Grand Unification Theories (GUT).

*antoshkinalig@gmail.com, Work supported by RFBR project No. 14-22-03090 


\section{SEARCHING STRATEGIES IN NOVA}

The Dirac's electric charge quantization relation says $e \times g=n \times \frac{\hbar c}{2}$, where $e$ is a basic electric charge and $n$ is an integer. It means that magnetic monopoles could have a magnetic charge $(g) 68.5$ times greater than the charge of the electron. As the result they are expected to be very highly ionizing (See Fig. 1 and [2]). For example, for $\beta=10^{-3}$ one can expect ionisation 10 times larger then for the cosmic muon. We call this muon MIP (Minimum ionizing particle) due to it's mean energy loss rate through matter.

"Slow" monopoles with $\beta<10^{-2}$ and less can be identified due to their linear tracks with long transit times through the detector (See Fig. 2). In this figure one can see a software visualization of the Far detector and the monopole in it's center. The monopole's energy in this case is almost at the ADC saturation limit (12 bits - 4096 counts). In terms of energy it is more then $650 \mathrm{MeV}$.

Monopoles with $\beta=10^{-3}$ take $5 \mu$ s to cross the whole detector (one can see the temporal distribution on the bottom of Fig. 2) in comparison with cosmic muons, which take only $50 \mathrm{~ns}$.

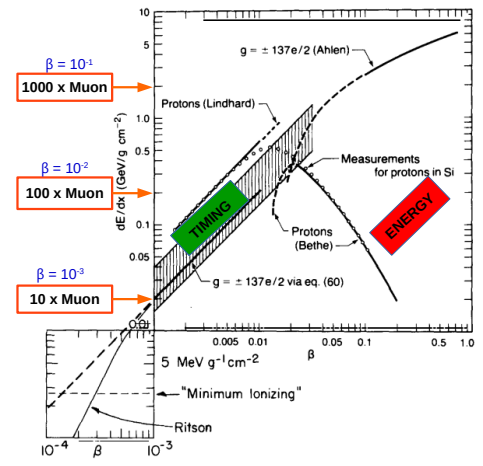

Figure 1. Monopole ionization [2]

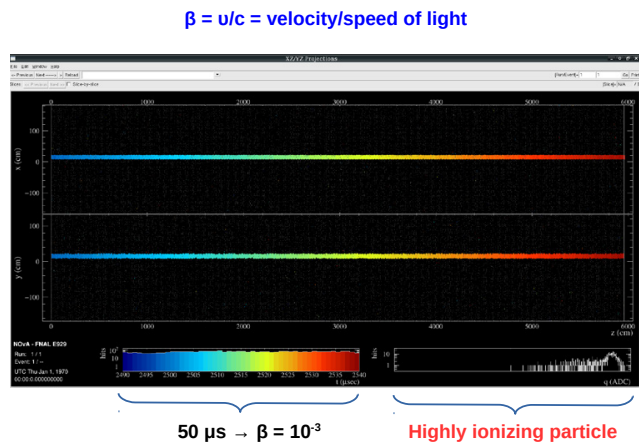

Figure 2. Event display with simulated monopole

In order to proceed to actual measurements using NOvA setup one needs to determine whether it is possible to achieve sufficient sensitivity first. Ordinary signals in NOvA detectors have time width less than $100 \mathrm{~ns}$ and energy less than $300 \mathrm{ADC}$ counts. Actual tests could be performed utilizing the NOvA test bench at JINR.

\section{TEST BENCH AT JINR AND IT'S ROLE}

NOvA test bench @JINR consists of a few components. First is the native NOvA electronics: Avalanche PhotoDiode and Front-End Board. Second is the special hardware: download cable, DCM-emulator, LED, Low and High Voltage Sources, Pulse generator and cooling system. Third is a PC with necessary software.

All devices were put into a black metal box. The black box allows one to perform all the measurements with photodetectors like APDs and PMTs, and screens all external electromagnetic noises. 
A pulse generator waits for the FEB trigger and sends the electric pulse to the LED. Then the light pulse from the LED comes through the fibers to the APD, and the FEB is reading at this moment (See Fig. 3).

Measurements performed on this bench demonstrated that it is possible to use NOvA electronics for the search of monopoles. It means that one can see long signals $(5 \mu s)$ with high energy distributions and reconstruct them with high efficiency. For more details see [7].

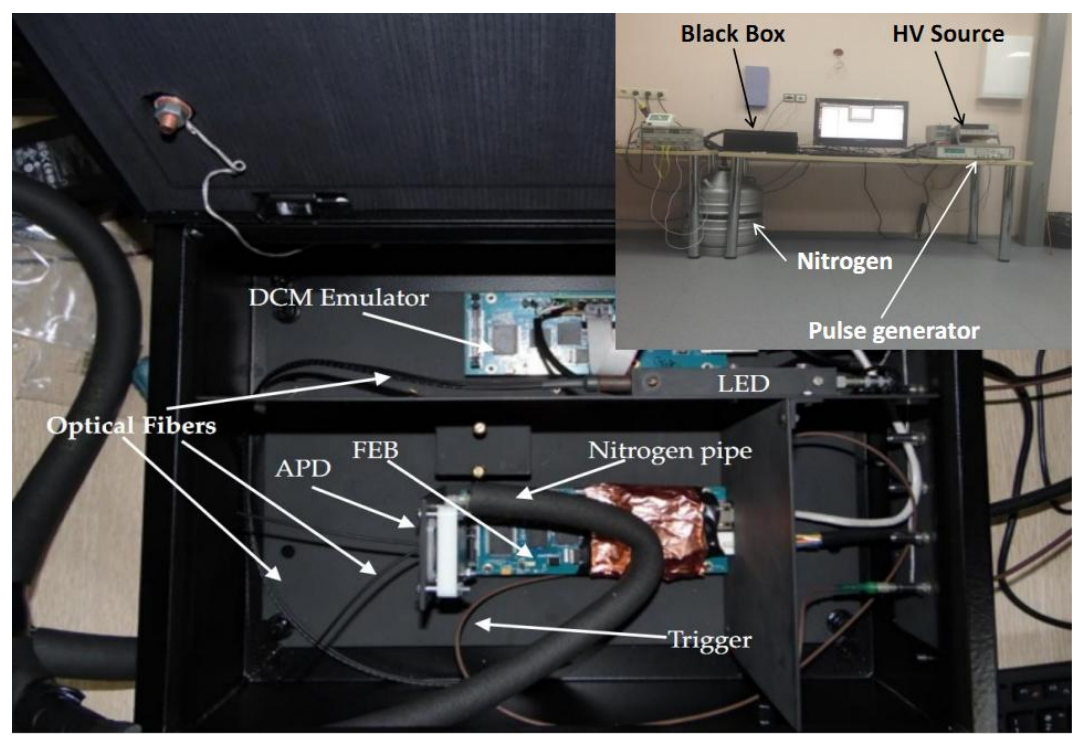

Figure 3. NOvA test bench at JINR

\section{SLOW MONOPOLE TRIGGER}

The slow monopole trigger was implemented in June 2015. It allows for the identification of slow ( $\beta<10^{-2}$ and less) monopole tracks by checking the number of plane gaps between the entry and exit hits. We look at all of the hit planes in the contained area and look for gaps. This trigger helps us to choose primary candidates for our further analysis.

\section{OFFLINE RECONSTRUCTION}

We have a special procedure for "catching" (See Fig. 4) a monopole track in the real data. Basically the algorithm proceeds by first removing all of the relativistic cosmic rays to form a single monopole cluster. This cluster is then divided into several monopole slices. Finally, we look for track-like objects in the monopole slices and form monopole tracks.

The tracks are then sorted from slowest to fastest track. In other words, the first track is the slowest track. The current reconstruction implementation retains the information of the three slowest tracks for each event. Generally, we expect only one track per event, but this algorithm may also identify several tracks in one event. 


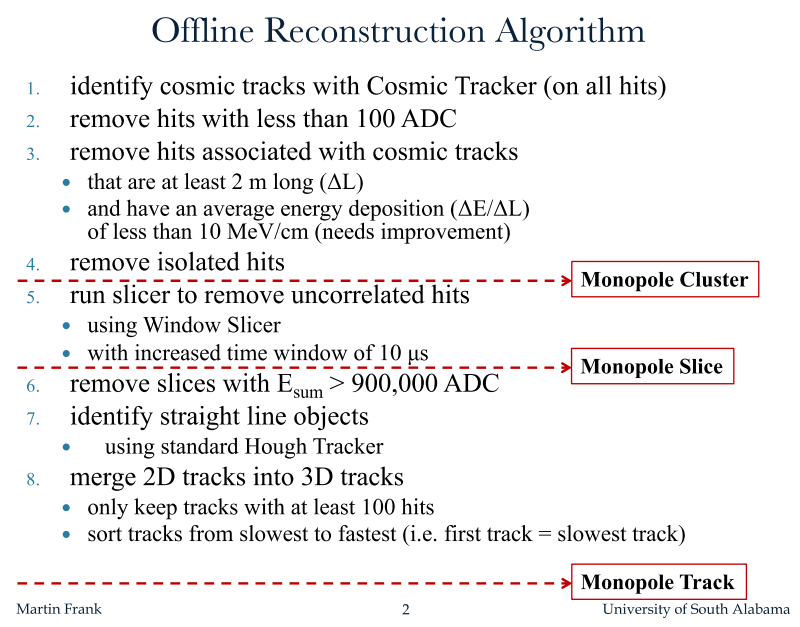

Figure 4. "Catching" algorithm

\section{Data Samples}

We have several datasets for testing of our algorithm:

Monte Carlo (Simulated monopoles $+5 \mathrm{~ms}$ long non-bias data produced by the daily SNEWS trigger - true monopole and nominal detector activity). Four velocities $\beta_{\text {sim }}$ : $5 \times 10^{-4}, 1 \times 10^{-3}, 5 \times 10^{-3}, 1 \times 10^{-2}$.

Slow Monopole Triggered Events (Slow Monopole Trigger - first run is 19728, last one is 20752 for Low Gain (100) and 20753 like the first one for the new Data Set with High Gain (150).) These numbers (100 and 150) refer to the APD gain.

\section{FURTHER ALGORITHM AND PRELIMINARY RESULTS}

Currently we have tested 10\% of the Low Gain data. Our main tools for post selection are: Linear Regression coefficient and Time Gap Fraction.

\section{Linear Regression coefficient}

We fit a line to the collection of hits associated with the monopole track. Using standard linear regression techniques (see [8]), we can calculate the squared regression coefficient $\left(r^{2}\right)$ for hits in the $x t$ and $y t$ spaces separately. A value of $r^{2}$ equal to unity means that the hits lie on a straight line while a value of zero means that the hits are uncorrelated. For a high-quality track, we require the linear regression coefficient to be close to unity. This calculation is performed separately for each view, yielding $r^{2}{ }_{x t}$ and $r_{y t}^{2}$ for each track. The minimum of these two quantities is called $r^{2}{ }_{\min }$. Therefore, if we require $r^{2}{ }_{\min }$ to have a large value close to unity, both $r_{x t}^{2}$ and $r_{y t}^{2}$ will have to exceed this requirement.

\section{Time Gap Fraction}

First, we sort all of the hits associated with the monopole track by time. We then look for the largest gap in time between adjacent hits $\left(\delta t_{\max }\right)$. The time gap fraction is then

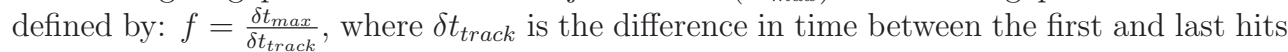
of the monopole track sorted by time. A high-quality track will have a value of $f$ close 
to zero indicating that there are no gaps in time along the track. A track that consists of two independent clusters of hits will have a value of $f$ close to unity. We therefore require $f$ to be close to zero. This calculation is performed separately for each view, yielding $f_{x t}$ and $f_{y t}$ for each track. The maximum of these two quantities is called $f_{\max }$. Therefore, if we require $f_{\max }$ to have a small value close to zero, both $f_{x t}$ and $f_{y t}$ will have to be below this requirement.

\section{Track Requirements}

For a track to be linear, it has to meet the following requirements: $r^{2}{ }_{\min } \geq 0.95$ and $f_{\max } \leq 0.2$. We consider an event linear if its primary monopole track is linear.

Right now we observed no slow events in the $10 \%$ data sample.

\section{CONCLUSION}

Our research shows that it is possible to measure lighter monopoles on NOvA far detector. The detector itself has the unique potential to "touch" a new region of phase space due to it's location on the surface and large surface area. These factors give one very high chance to "catch" the magnetic monopoles, or make the limit on the monopole flux (see [6]). Preliminary calculation gives one upper flux limit $\Phi=4.1 \times 10^{-15} \mathrm{~cm}^{-2} \mathrm{~s}^{-1} \mathrm{sr}^{-1}$.

The Slow Monopole Trigger is proved to provide sufficient precision. A special "cutter" and "selector" were developed. We tested only $10 \%$ of the Low Gain data. A technote about the general search strategy and description of tools is ready (see [9]) and right now we have asked to allow us apply the "cutter" for the remaining data. The collaboration gave us useful comments and we solved the majority of the issues and shortcomings. We still do not see any good candidates in real data, but our cutter and selector work well on Monte Carlo events.

\section{ACKNOWLEDGMENTS}

We would like to thank the NOvA Collaboration for providing us with unpublished and public information.

\section{REFERENCES}

[1] Martin Frank, 2017 SESAPS NOvA Slow Monopole Talk, http://nova-docdb.fnal.gov/cgibin/ShowDocument?docid $=24903$

[2] Donald E. GROOM, In search of the supermassive magnetic monopole, PHYSICS REPORTS (Review Section of Physics Letters) 140. No. 6 (1986) 323373. North-Holland, Amsterdam

[3] Alexander Antoshkin et all., NOVA test bench at JINR http://nova-docdb.fnal.gov/cgibin/ShowDocument?docid $=14987$

[4] Site of the NOvA experiment. URL: https://www-nova.fnal.gov/

[5] NOvA Public Document Database, http://nova-docdb.fnal.gov/cgi-bin/DocumentDatabase

[6] Enhao Song, Monopole trigger efficiency and upper flux limit, NOvA DocDB - 15938-v1 (unpublished)

[7] Alexander Antoshkin, NOvA DocDB - 14261-v1 (unpublished).

[8] D. A. McQuarrie, Mathematical Methods for Scientists and Engineers, University Science Books (2003).

[9] Martin Frank et al., NOvA DocDB - 22610-v2 (unpublished). 